\title{
Conocimientos, actitudes y prácticas de los profesionales de la salud ante el VIH/SIDA en el primer nivel de atención
}

DOI: $10.46932 / \mathrm{sfjdv} 1 \mathrm{n} 4-006$

Received in: August 1st, 2020

Accepted in: September 30th, 2020

\section{Oscar Alejandro Moreno Solís}

Higher academic background: Médico Familiar

Current Institution: Servicio de Atención Familiar. Unidad Médica Familiar 16, Altamira Tamaulipas, Instituto Mexicano del Seguro Social, México.

Full address (can be institutional or personal, as you prefer): Calle 2 N/A, Jardines de Altamira,

Residencial Centro 1, 89600 Altamira, Tamaulipas.

E-mail: oams87@gmail.com

\section{Liliana Aurora Carrillo Aguiar}

Higher academic background: Médico Familiar., Master en Ciencias Médicas., Profesor Titular de la Residencia en Medicina Familiar.

Current Institution: Unidad Médica Familiar 77, Madero Tamaulipas, Instituto Mexicano del Seguro Social, México.

Full address (can be institutional or personal, as you prefer): Blvd. Adolfo López Mateos S/N, Col. Las Conchitas, Madero Tamaulipas.

E-mail: lilifha@ hotmail.com

\section{Brian González Pérez}

Higher academic background: Médico Familiar., Master en Ciencias Médicas., Master en Innovación y

Tecnología Educativa., Profesor Investigador.

Current Institution: Servicio de Atención Familiar. Unidad Médica Familiar 38, Tampico Tamaulipas, Instituto Mexicano del Seguro Social, México. Facultad de Medicina "Dr. Alberto Romo Caballero"

Universidad Autónoma de Tamaulipas, Tampico, Tamaulipas, México.

Full address (can be institutional or personal, as you prefer): Calle Argentina y Canadá 107 Col. Infonavit C.P. 89310, Tampico, Tamaulipas.

E-mail: brian.gonzalez.perez@gmail.com

\section{Ricardo Salas Flores}

Higher academic background: Endocrinólogo Pediatra., Master en Ciencias Médicas., Profesor Investigador.

Current Institution: Coordinación de Educación en Salud e Investigación. Hospital General Regional No. 6, Madero, Tamaulipas, Instituto Mexicano del Seguro Social, México. Facultad de Medicina "Dr.

Alberto Romo Caballero" Universidad Autónoma de Tamaulipas, Tampico, Tamaulipas, México. Full address (can be institutional or personal, as you prefer): Blvd. Adolfo López Mateos S/N, Col. Las Conchitas, Madero Tamaulipas.

E-mail: risafl@yahoo.com 
Verónica Olvera Mendoza

Higher academic background. Master en Finanzas., Profesor Investigador.

Current Institution: Facultad de Medicina "Dr. Alberto Romo Caballero" Universidad Autónoma de Tamaulipas, Tampico, Tamaulipas, México.

Full address (can be institutional or personal, as you prefer): Centro Universitario Sur, Cto. Interior S/N CP 89337, Tampico, Tamaulipas

E-mail:volvera@docentes.uat.edu.mx

\section{RESUMEN}

INTRODUCCION: El Virus de inmunodeficiencia humana (VIH) / Síndrome de inmunodeficiencia adquirida (SIDA) es una enfermedad infectocontagiosa que a pesar de ser prevenible, sigue siendo un importante problema de salud pública a nivel mundial y sus portadores padecen el riesgo de sufrir prejuicios y discriminación por parte de la sociedad e incluso del mismo personal de salud, lo cual obstaculiza la correcta atención médica.

OBJETIVO: Evaluar los conocimientos, actitudes y prácticas ante el VIH/SIDA de los profesionales de la salud en el primer nivel de atención.

METODOS: Se realizó un estudio observacional, transversal, descriptivo y prolectivo. Se incluyó a personal de la salud de las áreas de medicina familiar, enfermería, estomatología y laboratorista de la Unidad de Medicina Familiar No. 77 del Instituto Mexicano del Seguro Social que aceptaron contestar la encuesta CAP ante el VIH/SIDA, la cual tiene tres dominios: Conocimientos, que incluye ítems sobre infecciones de transmisión sexual, formas de prevención, vías y fuentes de transmisión; Actitudes, que indaga por conductas de estigma, discriminación, rechazo y Prácticas de riesgo para adquirir la infección.

RESULTADOS: Se encuestaron un total de 81 trabajadores, 61 mujeres y 20 hombres; con rango de edades entre 23 a 60 años, con una media de 42.47 + 7.7 años. Las categorías fueron: 50 médicos, 15 laboratoristas, 10 enfermeras y 6 estomatólogos. Se encontró que las enfermeras obtuvieron mayor porcentaje correcto de respuestas en el dominio de Conocimientos (83.6\%), los médicos en cuanto a Actitudes $(42.1 \%)$ y los estomatólogos en el dominio de las Prácticas (61.1\%). No hubo diferencias al comparar los porcentajes de respuestas correctas según el sexo $(p=0,25)$, ni edad $(p=0,89)$, así como en la relación entre los tres dominios $(\mathrm{p}=0.874)$.

CONCLUSIONES: A pesar de que se tiene el conocimiento sobre el VIH/SIDA, aún se tiene cierto rechazo hacia la enfermedad, por lo que es importante reforzar los protocolos de atención médica.

Palabras-Clave: Prácticas profesionales, actitudes, virus de inmunodeficiencia humana, personal de salud, atención médica.

\section{INTRODUCCION}

El Virus de inmunodeficiencia humana (VIH)/Síndrome de inmunodeficiencia adquirida (SIDA) es una infección prácticamente de reciente descubrimiento, siendo el año de 1983 cuando se descubría que un retrovirus provocaba una infección crónica que después de un periodo de latencia termina por evidenciar un grave deterioro inmunológico gestado a través de largos años (1).

El reporte de la Declaración del 2012 del programa conjunto de la Organización de las Naciones Unidas para el VIH/SIDA habla que, a finales del año 2011, 34 millones de personas vivían con el VIH, durante el 2011 se produjeron 1.7 millones de muertes provocadas por causas relacionadas y se 
reportaron 2.5 millones de infecciones nuevas, incluyendo 330,000 niños (2). El Centro Nacional para la Prevención y el Control del VIH/SIDA (Censida) en el 2012 reportó 157529 casos acumulados de sida, afectando primordialmente a personas entre 15 y 44 años (3). En el Instituto Mexicano del Seguro Social se determinó en el año 2009 que el grupo más afectado son hombres de entre 25 y 44 años y que tienen una mortalidad de 12 por cada 100000 hombres (4).

Al hablar de VIH se debe tratar siempre todo lo referente a su forma de actuar, su morbilidad y mortalidad que actúan por tres vías: 1) inmunodeficiencia 2) daño directo a órganos blanco y 3) daño a estos órganos por la inflamación crónica producida por el mismo virus (5). Debido a las diversas complicaciones generadas de la infección, tan sólo en el año 2002 generó un gasto de \$ 98, 614,832 pesos mexicanos tan sólo en el IMSS (6).

El VIH puede transmitirse a los individuos que reciben transfusiones de sangre contaminada con dicho virus, hemoderivados o trasplantes hísticos, así como los usuarios de drogas intravenosas expuestos al VIH (7). Existe un factor de riesgo que es la ventana serológica; ésta es el período inmediatamente después de ocurrir la infección, en el cual, el paciente está infectado, pero no presenta la sintomatología y no puede ser detectada por las pruebas de laboratorio (8).

Existe un riesgo laboral pequeño pero claro de transmisión del VIH entre los profesionales sanitarios, el personal de laboratorio y, posiblemente, de otras personas que manipulan las muestras de los pacientes con infección por el VIH. En Estados Unidos se calcula que entre 600000 y 800000 profesionales sanitarios sufren cada año pinchazos con las agujas u otros instrumentos punzantes de uso médico. En la actualidad, todas las heridas por punción y las exposiciones mucosas en los trabajadores de la salud reciben tratamiento antirretroviral profiláctico (9).

La estrategia mundial del Sector de la Salud contra el VIH (2016-2021) describe diversas medidas de acción acelerada para agilizar e intensificar la respuesta frente al VIH y así cumplir el ambicioso objetivo de la ONU de poner fin al SIDA para el 2030 (10). Sin embargo el lograr un objetivo tan grande requiere de realizar muchos cambios en cuanto a infraestructura, aplicación de programas de prevención, generar cambios tangibles en la sociedad y también el redoblar esfuerzos del personal de salud, el tener un personal capaz, actualizado en el tema y que rija su práctica diaria encaminado hacia una correcta aplicación de las directrices marcadas por la ONU, las guías de práctica clínica (guía de prevención y tratamiento de ITS y Diagnóstico y Referencia oportuna del paciente con infección por el VIH en el primer nivel) y organizaciones de la salud de cada país; todo esto para que al final del día tengan disponible la misma información los médicos de cualquier parte del mundo. 


\section{ENCUESTA CAP}

Partiendo del hecho que debemos evaluarnos como profesional de salud con respecto a un tema en específico podemos utilizar el instrumento llamado CAP (Conocimiento, Actitudes y Prácticas) el cual puede ser utilizado en prácticamente cualquier tema de interés. Las preguntas CAP tienden a evidenciar rasgos característicos de los saberes, actitudes y comportamientos en materia de sanidad asociados a factores religiosos, sociales o tradicionales, pero también la concepción que cada persona toma acerca de su cuerpo o de la enfermedad investigada; esto nos puede dar la respuesta para conocer los frenos encontrados para el cambio que estamos buscando generar. Además, nos permite conocer la congruencia entre el conocimiento, la opinión y el actuar del entrevistado (11).

Es fundamental que el personal de salud tenga conocimiento acerca de la enfermedad VIH/SIDA para enfocarse en la prevención y diagnóstico de personas que se encuentren en riesgo de contraerla. Es preciso contar con un conocimiento basado en guías actualizadas para tener la seguridad de transmitir información valiosa al paciente y tener un resultado favorable en la lucha contra la transmisión de la enfermedad (12). Hay que recordar que el conocimiento será siempre el que nos ayuda a su vez contra la desinformación e ignorancia que resultan en una actitud negativa al tratar con pacientes seropositivos. El presente trabajo tiene como objetivo evaluar los conocimientos, actitudes y prácticas ante el VIH/SIDA de los profesionales de la salud de la Unidad Médica Familiar N ${ }^{\circ} 77$ del IMSS. Esto a su vez permitirá conocer las áreas a reforzar del personal hacia la enfermedad y mejorar así la atención a este sector de la población afectado por la enfermedad y marginado por la sociedad.

\section{METODOS}

Se realizó un estudio observacional, transversal, descriptivo y prolectivo autorizado por el Comité Local de Investigación en Salud 2804 del Instituto Mexicano del Seguro Social durante el periodo comprendido de enero a marzo 2019. Se incluyó al personal de la salud de cualquier edad, sexo y turno laboral de las áreas de medicina familiar, enfermería, odontología y laboratorista adscritos a la Unidad de Medicina Familiar No. 77 del IMSS, que tengan contacto con pacientes y manipulen líquidos, fluidos corporales o excretas de pacientes portadores de VIH/SIDA. En caso de cumplir con los requisitos, se le solicitó su participación voluntaria explicando de manera inteligible el concepto, objetivos y requerimientos de la investigación, junto a las dudas que pudieran surgir. Los que aceptaron participar en el estudio se les solicitó firmar un consentimiento informado estandarizado para los proyectos de investigación con clave 2810-009-013, en el cual, se exponen los puntos de ética y confidencialidad de los datos proporcionados. Una vez terminado el proceso de selección, se recolectaron los parámetros necesarios por medio de una encuesta CAP ante el VIH/SIDA, la cual tiene 
tres dominios: Conocimientos, que incluye ítems sobre infecciones de transmisión sexual, formas de prevención, vías y fuentes de transmisión; Actitudes, que indaga por conductas de estigma, discriminación, rechazo y Prácticas de riesgo para adquirir la infección.

Los datos recolectados se codificaron para protección de la información de los pacientes y tuvieron acceso a la base de datos solamente los propios investigadores. La base de datos y el análisis se hicieron con ayuda del paquete estadístico Statistical Package for Social Sciences para Windows (versión 19; SPSS Inc., Chicago IL, USA). Para el análisis de los resultados se utilizó estadística descriptiva (medidas de tendencia central y de dispersión), estadística inferencial (pruebas paramétricas y no paramétricas, según la normalidad o no normalidad de los resultados, con prueba de Kolmogorov Smirnov; análisis de varianza o Kruskal Wallis, Coeficiente de correlación de Pearson o Spearman, así como T de Student para muestras independientes o Wilcoxon), considerando significancia estadística con valores de $\mathrm{p}<0.05$.

\section{RESULTADOS}

Se encuestaron un total de 81 trabajadores, 61 mujeres y 20 hombres; con rango de edades entre 23 a 60 años, con una media de 42.47 + 7.7 años. Las categorías fueron: 50 médicos, 15 laboratoristas, 10 enfermeras y 6 odontólogos. Se encontró que las enfermeras obtuvieron mayor porcentaje correcto de respuestas en el dominio de Conocimientos (83.6\%), los médicos en cuanto a Actitudes (42.1\%) y los estomatólogos en el dominio de las Prácticas (61.1\%) (Gráfico 1).

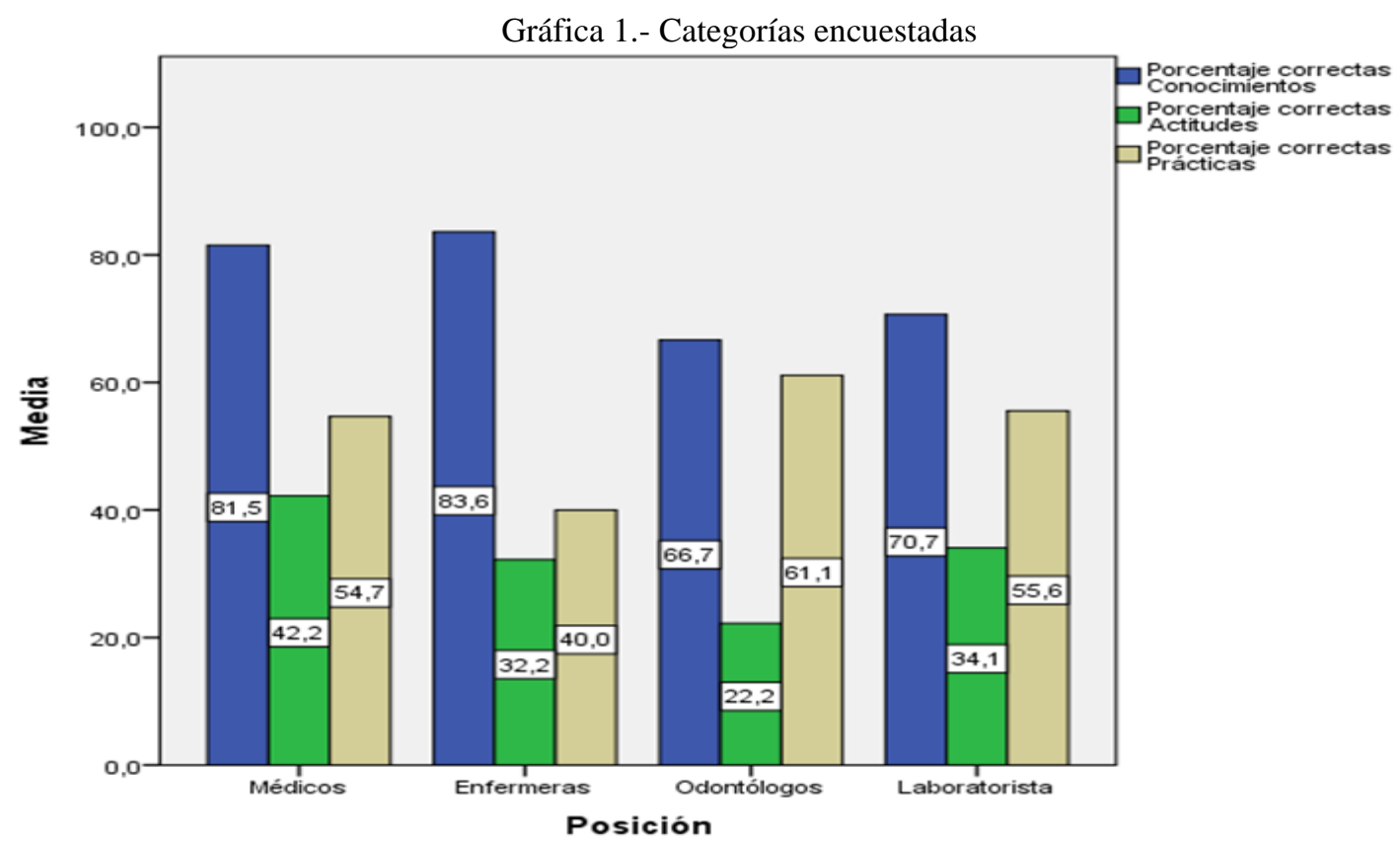

No hubo diferencias al comparar los porcentajes de respuestas correctas según el sexo ( $\mathrm{p}=0,25)$, ni edad ( $\mathrm{p}=0$,89), así como en la relación entre los tres dominios $(\mathrm{p}=0.874)$, (Gráfico 2). 


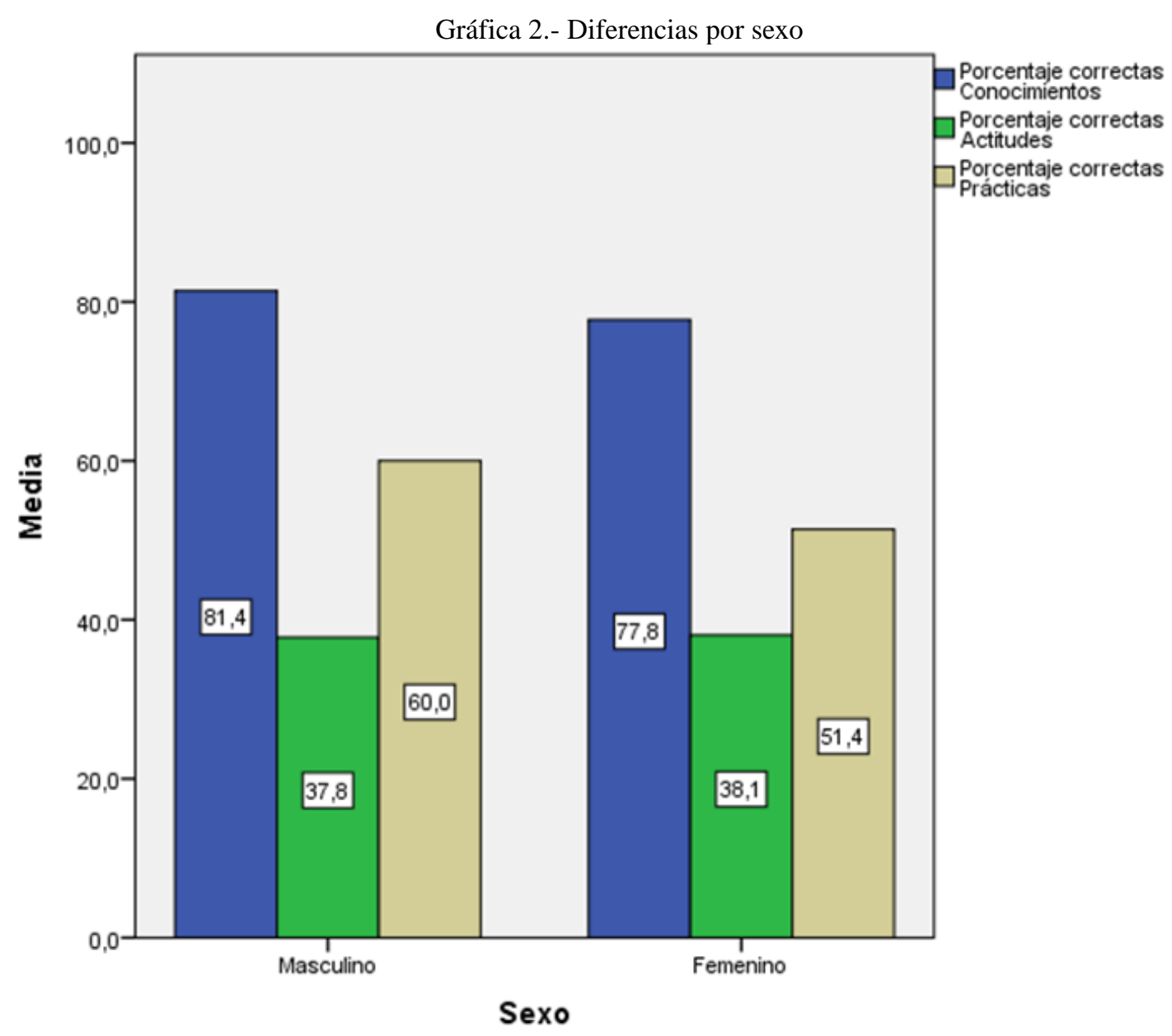

\section{DISCUSIÓN}

Las investigaciones utilizando un instrumento tipo CAP aunque no son nuevas, aún no se obtiene un máximo aprovechamiento de este valioso recurso, por lo cual en nuestro país aún tenemos pocas de investigaciones de este tipo acerca del VIH para profesionales de la salud. Sería interesante realizar este tipo de investigación en nuestro primer nivel de atención antes y posterior a un curso de actualización acerca del paciente y enfermedad VIH/SIDA, lo cual nos ayudaría a realizar actualizaciones del tema mucho mejor orientadas de lo que se podría obtener con las limitantes de un estudio meramente transversal. Muestra de esto queda plasmado en un estudio ESTVIH español realizado a profesionales de medicina y enfermería de atención primaria de la comunidad en Madrid donde mediante una encuesta CAP online pre y post-implementación de nuevas estrategias se logró mejorar la detección precoz de VIH/SIDA (13).

En el presente estudio obtuvimos un $78.66 \%$ de respuestas correctas del personal encuestado en el ámbito de conocimientos, lo cual es un indicador de que se tienen buenos conceptos clave acerca de la enfermedad, de su prevención y diagnóstico oportuno, contrario a lo que naturalmente se pensaría, las 
enfermeras fueron las que tuvieron una puntuación promedio mayor $(83,6 \%)$ que incluso los médicos y fueron los odontólogos los que tuvieron un desempeño menor con 66,7\%.

En un estudio CAP sobre el VIH/SIDA realizado a médicos docentes de la Facultad de medicina, Universidad Mayor de San Simón, se obtuvo un promedio de respuestas correctas de $61,6 \%$ lo cual mostró que en el primer nivel de atención analizado se tenía un mejor nivel de conocimientos que entre los profesores de la facultad (14).

En el global de la sección de actitudes se obtuvo un 37,99\% siendo ésta la sección de la encuesta en la que se obtuvo un menor aprovechamiento, la categoría con el promedio más alto fue la de los médicos con un 42,2\% y la que obtuvo el menor desempeño fue la de odontología con 22,2\%. Este resultado sugiere que nuestro personal de salud aún presenta cierta resistencia hacia este tipo de pacientes o la enfermedad en sí.

Haciendo la comparativa con el mismo estudio anterior nuestro primer nivel se queda muy debajo de su $92 \%$ de respuestas correctas en esta sección, sin embargo, podríamos hacer énfasis a que desconocemos qué porcentaje de los docentes encuestados tienen una práctica profesional que los relacione con pacientes seropositivos.

En la sección de prácticas se obtuvo una media de 53,49\% siendo los odontólogos los que obtuvieron un promedio mayor con $61,1 \%$ y las enfermeras las que obtuvieron el menor con $40,0 \%$. Este resultado sería una razón para promover cursos de actualización en el tema y el proceder del personal ante la detección de casos o la sospecha de estos. Nuestro estudio tiene una puntuación similar al obtenido por un estudio CAP colombiano el cual obtuvo un puntaje de 58,8\% en el apartado de prácticas, lo cual nos pone en un mismo nivel que los profesionales de salud evaluados en éste estudio (15).

Se debe dar a conocer a la población las vías y mecanismos de transmisión, formas de prevención y servicios de información, detección oportuna y atención integral. El sistema interactivo de Wandersman tiene como bases para la transferencia del conocimiento: la síntesis y sistema de transferencia, un sistema de apoyo y un sistema de entrega (16). En países que son mayormente afectados por el VIH/SIDA, como Nigeria, ven hoy en día a las escuelas y los sistemas de salud como una oportunidad para llevar a los jóvenes educación de prevención antes de que sean sexualmente activos (17). Se debe fomentar en los pacientes con VIH/SIDA el auto cuidado de la salud incluyendo medidas de prevención como sexo seguro y sexo protegido para romper la cadena de infección y reinfección (18). 


\section{CONCLUSIONES}

Del personal encuestado podemos concluir que estamos ante un personal que tiene conocimientos clave de la enfermedad, el área de conocimientos es lo que nos arroja. Sin embargo, éste conocimiento no se observa a la par en cuanto a las actitudes y prácticas que debe tener el personal de salud que está en interacción con este tipo de pacientes.

Como personal de salud de primer nivel aún tenemos rechazo a la enfermedad y al paciente portador de VIH/SIDA cuando al ser una enfermedad bien conocida y estudiada a través de décadas, tendríamos que poder hablarla más abiertamente con el paciente y no mostrar ningún tipo de diferencias en nuestro trato. Es importante que el personal de salud conozca el protocolo de estudio de un paciente con diagnóstico de VIH para que así en su práctica diaria sepa cómo actuar ante un caso detectado. El llevar cursos de actualización sería una buena manera de reforzar el conocimiento y fomentar una buena práctica con el paciente, asimismo sería interesante continuar realizando investigaciones futuras de este tema para valorar el progreso que tenga el personal en su práctica diaria.

\section{REFERENCIAS}

1.-Iglesias CM , Reyes G, Terán RM, Ortiz MM. Treinta años del VIH-SIDA: perspectivas desde México. 2015. México D.F.: Histórica.

2.-Organización Pan Americana de la Salud. Protocolo Integrado de VIH e ITS en Atención Primaria. Recuperado de http://www.paho.org/par/index.php?option=com_docman\&view=download\&category_slug=epidemiolo gia-y-control-de-enfermedades\&alias=492-protocolo-8-manual-de-aps-para-el-manejo-de-vih-eits\&Itemid $=253$

3.-Rodríguez CD, Magis RC, Bravo GE. Barreras que afectan el uso de datos de vigilancia epidemiológica del VIH/sida en México. Sal Pub Mex, 2013; 55(4):408-415.

4.-José Luis Vázquez-Martínez, María Guadalupe Mercadillo-Pérez, Juan Germán Celis-Quintal. (2011). Mortalidad por VIH/SIDA en el Instituto Mexicano del Seguro Social. Rev Med Inst Mex Seguro Soc, 49 (1), 109-115

5.-Censida/Secretaría de Salud Guía de manejo antirretroviral de las personas con VIH. México, Sexta edición. 2014. Recuperado de www.censida.salud.gob.mx/descargas/principal/Guia_ARV_2014V8.pdf

6.-Zambrana MB, Ramírez TJ, Coria I. Gasto hospitalario de cinco patologías de alto impacto económico. Rev Med Inst Mex Seguro Soc, 2008;46(1):43-50. 
7.- Longo DL, Kasper DL, Jameson J, Fauci AS, Hauser SL, Loscalzo J. (2012). Harrison Principios de Medicina Interna. México: McGraw-Hill; 2012.

8.- Suárez G, Eranilde L, De Freitas F, Henry A, Hannaoui R, Erika J, Gómez A. Prevalencia de enfermedades infecciosas de transmisión sanguínea en donantes que asisten al Banco de Sangre del Hospital Universitario “Antonio Patricio de Alcalá, Cumaná, Estado Sucre. Kasmera [Internet]. 2007;35(1): 56-64. Disponible en: http://www.scielo.org.ve/scielo.php?script=sci_arttext\&pid=S0075$52222007000100007 \& \operatorname{lng}=$ es.

9.- Gopar NR, Juárez PC, Cabello LA, Haro GL, Aguilar MG. Panorama de heridas por objetos punzocortantes en trabajadores intrahospitalarios. Rev Med Inst Mex Seguro Soc 2014;53(3):356-61.

10.- World Health Organization. Estrategia mundial del sector de la salud contra el VIH, 2016-2021: OMS. [Internet]. [Consultado 2016]. Disponible en: http://apps.who.int/iris/bitstream/handle/10665/250574/WHO-HIV-2016.05spa.pdf;jsessionid=0B21C2E4F227B0DC9176173453EB8151? sequence=1

11.- Gumucio. S. Recogida de Datos. Métodos cuantitativos. Ejemplos de encuestas CAP: Medicins du Monde. [Internet]. [Consultado 2001]. Disponible en: https://issuu.com/medecinsdumonde/docs/mdm_guia_encuesta_cap_2011

12.- Dirección General de Epidemiología/Secretaría de Salud. Manual de Procedimientos Estandarizados para la Vigilancia Epidemiológica del VIH-SIDA.2012. Obtenido de http://www.censida.salud.gob.mx/descargas/biblioteca/documentos/Manual_VIHSIDA_vFinal_1nov12. pdf.

13.- Dirección General de Salud Pública Madrid (2017). Conocimientos, actitudes y prácticas de profesionales de atención primaria sobre diagnóstico precoz de VIH: encuesta pre-post intervención ESTVIH.

14.- Valdéz E., Avilés J. \& Saudán A. Conocimientos, actitudes y práctica sobre el VIH/sida en docentes de la Facultad de Medicina, Universidad Mayor de San Simón. Gac Med Bol 2012; 35 (1): 22 26.

15.- Uribe AF, Orcasita LT. Evaluación de conocimientos, actitudes, susceptibilidad y autoeficacia frente al VIH/sida en profesionales de la salud. Av Enferm 2011;29(2):271-284.

16.- Olvera BM, Moreno MM, Garza SS. Transferencia del conocimiento para la prevención de ITS/VIH/SIDA en adolescentes. Rev Med Inst Mex Seguro Soc, 2014;53(6):742-748.

17.- Abdu M, Umar A, Hj B, Faisal I, Syed H, Syed T y cols. Effectiveness of HIV/AIDS educational intervention in increasing knowledge, attitude and practices for primary school teachers in some part of Africa, HIV \& AIDS Review, 2016; 15:17-25.

18.- NORMA Oficial Mexicana NOM-010-SSA2-2010, Para la prevención y el control de la infección por Virus de la Inmunodeficiencia Humana. México. 2010. 